This is a postprint version of the following published document:

Loayza, A., Cabanelas, J. C., González, M. G. \& Baselga, J. (2015). Critical examination of chemically modified hybrid thermosets: Synthesis, characterization and mechanical behavior in the plateau regime of polyaminosiloxane-nitrileDGEBA. Polymer, 69, pp. 178-185.

DOI: 10.1016/j.polymer.2015.05.054

(C) Elsevier, 2015

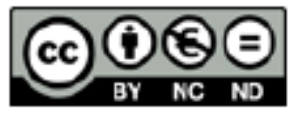

This work is licensed under a Creative Commons Attribution-NonCommercialNoDerivatives 4.0 International License. 


\title{
Critical examination of chemically modified hybrid thermosets: Synthesis, characterization and mechanical behavior in the plateau regime of polyaminosiloxane-nitrile-DGEBA
}

\author{
Artemia Loayza, Juan C. Cabanelas, María González, Juan Baselga* \\ Department of Materials Science and Engineering and Chemical Engineering, Universidad Carlos III de Madrid, Av. Universidad 30, 28911 Leganés, Madrid, Spain \\ * Corresponding author. mail address: jbaselga@ing.uc3m.es (J. Baselga).
}

\begin{abstract}
Poly(3-aminopropylmethylsiloxane) has been modified with acrylonitrile via aza Michael addition and a broad range of modified oligomers have been prepared with $\mathrm{CN}: \mathrm{NH}_{2}$ ratio ranging between 0.1 and 1 . NMR and FTnIR analysis reveals that acrylonitrile modification proceeds without formation of tertiary amines. Modified oligomers have been characterized by

DSC and analysis of Tg reveals that the adducts are self-associated probably due to weak hydrogen bonding and dipole interactions. The modified oligomers with a modification degree higher than 0.4 were miscible with DGEBA. The low and

high temperature relaxations of the cured thermosets have been measured by DMTA. In addition to the commonly observed $\beta_{2}$ relaxation, a new $\beta 1$ process linked by an isosbestic point to $\beta_{2}$ has been found. $\beta 1$ is attributed to an extended segment comprising the pendant propionitrile group as well as the ami-nopropyl segment that connects tertiary amines to the polysiloxane backbone. Elastic modulus as well as the $\alpha$ relaxation can be tuned from high $\mathrm{Tg}$ and high rubbery modulus to low $\mathrm{Tg}$ and high damping thermosets changing the nitrile content. The experimental network structure obtained from elastic measurements and the $\mathrm{Tg}$ were related through well established structure-property relations.
\end{abstract}

KEYWORDS

Epoxy resins

Hybrid thermoset

Polysiloxane

\section{Introduction}

The structure and properties of epoxy/siloxane reactive blends is currently an open question in the field of advanced thermosetting materials. Both components are immiscible and phase segregation limits the morphology and phase composition, size and distribution. Several efforts to improve the compatibility by introducing reactive groups in the silicon-based component have been compiled in a recent review [1]. Common approaches include the functionalization of the inorganic siloxane backbone with reactive functional end-groups [2] (epoxy, amine, acrylate or isocyanate), the synthesis of siloxane block copolymers with a resin compatible segment [3] or the introduction of pendant reactive groups [4]. This last solution was adopted by our group in earlier reports on the synthesis of poly(3-aminopropylmethyl siloxane) (PAMS) and its curing with DGEBA based epoxy resins $[5,6]$.
Crosslinking agents for epoxy resins based on polyfunctional aminosiloxanes render very interesting polymer-polymer hybrid systems in which structure changes dramatically with both chemical conversion [5,6] and polymerization protocol [7]. They belong to the class of hybrid thermosetting blends and present a number of distinctive features. Contrary to conventional thermosets, a phase separated system consisting of PAMS spherical domains surrounded by the continuous epoxy matrix is formed upon mixing both reactants. In spite of their thermodynamic incompatibility, both components begin to react and, depending on the specific epoxy precursor and curing protocol, homogeneous (at the micron scale) or partially homogeneous thermosetting polymers are formed $[7,8]$. Another distinctive characteristic is related with their modification. They can be modified by physical mixing with conventional toughening agents, such as butadiene homo-and copolymers yielding a fine morphology [8]; but if a small amount of PMMA is used, a polymer with an extremely high affinity for the epoxy precursor, an aphron-like morphology is obtained in which the reactant mixture self organizes into polyhedral aphrons excluding the PMMA towards the interphase [9]. 
However, the most interesting feature is probably concerned with their high functionality which, at the same time, increases the Young's modulus in the rubbery state, increases hydrophobicity [10] and switches gelation towards lower conversion fixing the final morphology well before limiting conversion. This high functionality can be tuned reacting some of the pendant amino groups with molecules that may impart specific properties while the remaining provide enough crosslinking density to give high performance thermosets. An earlier successful attempt was done reacting some amino groups with 2,3-Epoxypropyl phenyl ether [11] aiming to delay gel conversion and to increase miscibility with DGEBA. However, the Tg and viscosity of the modified oligomers notably increased due to the bulkiness of the pendant group limiting the suitability of this approach.

In this work, we present a new alternative for modification of PAMS using the well-known aza-Michael addition reaction of aliphatic primary amines to acrylonitrile [12], which, as we will show, proceeds extremely well in this particular case yielding modified PAMS oligomers with highly polar cyano groups. Firstly we will present the synthesis and characterization of these adducts demonstrating the absence of double additions, i.e. the modified oligomers contain a controlled ratio between primary and secondary amines. Next, the adducts will be used to prepare a broad range of organo-inorganic thermosets differing in the amount of nitrile groups, i.e., different crosslinking densities, finding that for $\mathrm{CN}: \mathrm{NH}_{2}$ ratios higher than 0.4 , homogeneous systems at the micron scale are formed. The paper then focuses on the analysis and critical interpretation of the low and high temperature relaxations and in their elastic behavior. The high T relaxations will be explained using the concentration of elastically active network chains obtained from elastic measurements.

\section{Experimental part}

\subsection{Materials}

Diglycidyl ether of bisphenol-A (DGEBA, $M=377 \mathrm{~g} \mathrm{~mol}^{-1}$, $n=0.14$ ) and acrylonitrile (AN, 99\% purity) were purchased from Sigma-Aldrich. 3-aminopropylmethyldiethoxysilane (APDES) was purchased from ABCR Gmbh (97\% purity) and used without further purification.

\subsection{Synthesis and characterization of poly(3- aminopropylmethylsiloxane) precursor}

Poly(3-aminopropylmethylsiloxane) (PAMS) was synthesized form APDES by hydrolysis and condensation in aqueous solution according to methods already reported $[5,6,13]$. Molar mass and cyclics content were measured by MALDI-TOFF spectrometry (4700 Proteomics Analyzer, Applied Biosystems) with external calibration. Spectra were taken in reflector mode focusing on 1500 uma. Sample was dissolved in dicholoromethane $\left(1 \mathrm{mg} \mathrm{mL}^{-1}\right)$. Matrix solution was prepared with 2,5-dihydroxybenzoic acid (10 $\mathrm{mg} \mathrm{mL}^{-1}$ ) in ethanol: dichloromethane (25:75). Sample and matrix solutions were mixed (1:10 ratio) and $0.5 \mu \mathrm{L}$ aliquot was deposited onto the MALDI sample holder. Results show that PAMS consists of a mixture of linear and cyclic oligomers; cyclics amount $40.6 \%$ in number with number and weight average molar masses of $M_{n}(C)=744 \mathrm{~g} \mathrm{~mol}^{-1}$ and $M_{w}(C)=926 \mathrm{~g} \mathrm{~mol}^{-1}$. The number and weight average molar masses of the linear fraction are $M_{n}(L)=1250 \mathrm{~g} \mathrm{~mol}^{-1}$ and $M_{w}(C)=1508 \mathrm{~g} \mathrm{~mol}^{-1}$. The average molar masses of the sample are $M_{n}=1036 \mathrm{~g} \mathrm{~mol}^{-1}$ and $M_{w}=1343 \mathrm{~g} \mathrm{~mol}^{-1}$. The number and weight average polymerization degrees are $x_{n}=8.74, x_{w}=11.35$. Consequently, the average monomer molecular weight is $118.52 \mathrm{~g} \mathrm{~mol}^{-1}$.

\subsection{Synthesis and characterization of PAMS-acrylonitrile adducts}

PAMS-acrylonitrile adducts were synthesized via aza-Michael addition reaction (Scheme 1 ). Nine adducts were prepared with varying $\mathrm{CN}: \mathrm{NH}_{2}$ ratio from $10 \%$ to $100 \%$. Samples were designed as $\mathrm{Ax}$, where $\mathrm{x}$ is the nominal percentage of AN (Table S1 Supplementary material). In a typical experiment, adequate amounts of AN where added dropwise over $4 \mathrm{~g}$ of pure PAMS at room temperature $\left(23{ }^{\circ} \mathrm{C}\right)$ in a $25 \mathrm{~mL}$ double necked round flask continuously purged with a small $\mathrm{N}_{2}$ stream and with slight agitation. Temperature was measured all along the reaction (Fig. S1 Supplementary material); although reaction was completed in less than 20 min, agitation and $\mathrm{N}_{2}$ stream were maintained for $2 \mathrm{~h}$ and then the reaction mixture was vacuum distilled to remove AN traces and stored at $-4{ }^{\circ} \mathrm{C}$ in vacuum. Adducts characterization was done by FTIR (Perkin Elmer, GX2000) in both the medium (4000-400 $\mathrm{cm}^{-1}$ ) and near ranges $\left(7500-4000 \mathrm{~cm}^{-1}\right.$ ) (four scans, $\left.4 \mathrm{~cm}^{-1}\right)$, and by ${ }^{1} \mathrm{H}$ NMR (AVANCE DPX-300). ${ }^{1} \mathrm{H} \mathrm{NMR}\left(\mathrm{CDCl}_{3}, 298 \mathrm{~K}\right.$, $300 \mathrm{MHz}), \delta$ (ppm): C1 (0.47, m; 2H), C2 (1.44, m; 2H), C3 (2,61, m; $2 \mathrm{H}), \mathrm{C} 4(2.44, \mathrm{t}(\mathrm{br}) ; 2 \mathrm{H}), \mathrm{C} 5(2.83, \mathrm{t} ; 2 \mathrm{H})$.

\subsection{Curing of adducts with DGEBA}

For DMTA measurements, the following method was used to prepare specimens. Stoichiometric amounts of DGEBA and adducts were thoroughly mixed, vacuum degassed and introduced in a steel mold which was previously treated with a releasing agent (Frekote $\left.^{\circledR}\right)$. The mold was designed to obtain $25 \times 10 \times 2 \mathrm{~mm}^{3}$ specimens. Mold was heated up to $120^{\circ} \mathrm{C}$ in an oven for $2 \mathrm{~h}$ followed by a postcuring treatment at $140{ }^{\circ} \mathrm{C}$ for $30 \mathrm{~min}$. This curing protocol ensured full conversion as it was verified by the absence of residual heat in DSC and by the absence of residual epoxy and amine bands by FTIR. Samples were designated as ThAx.

Cured specimens were characterized by dynamic mechanical thermal analysis (DMTA, TA Instruments, Q 800, bending mode, 1, 10 and $30 \mathrm{~Hz}, 2^{\circ} \mathrm{C} \mathrm{min}^{-1}$ ). The high temperature relaxation, $\mathrm{T}_{\alpha}$, was measured at the maximum in $\tan \delta$ at $1 \mathrm{~Hz}$. Moduli data in the elastomeric state were obtained at $T=T_{\alpha}+50 \mathrm{~K}$.

Density measurements of the cured specimens were carried out in an He pycnometer (Micrometritics, AccPyc 1330) at $35.1^{\circ} \mathrm{C}$ with a typical standard deviation of $2 \mathrm{~kg} \mathrm{~m}^{-3}$. For calculation of the concentration of elastically active chains from modulus values at $\mathrm{Tg}$ $+50 \mathrm{~K}$, density data were corrected at that temperature using typical data for the linear thermal expansion coefficient, $\alpha_{L}$, below $\left(5010^{-6} \mathrm{~K}^{-1}\right)$ and above $\left(17010^{-6} \mathrm{~K}^{-1}\right)$ glass transition temperature for epoxy resins cured with amines [14], through the use of the following relation $\rho(T)=\rho\left(T_{0}\right) /\left[1+3 \alpha_{L} \Delta T\right]$. This correction decreased density data in $3-4 \%$, one order of magnitude higher than typical standard deviation of the measurement.

\section{Results and discussion}

\subsection{Synthesis and characterization of the adducts}

The aza-Michael reaction is a well-known method to conjugate amines to $\alpha, \beta$ unsaturated nitriles [15]. It usually requires basic conditions [16], such as the medium provided by the pure PAMS used here, and high conversions are usually achieved at moderate temperatures. The method used here does not use any solvent since PAMS is a low viscosity liquid in which AN readily dissolves, and we have found that the reaction is highly exothermic reaching almost completeness in a short period of time, typically less than $20 \mathrm{~min}$ (see Fig. S1 Supplementary material). The two main parameters of the synthesized adducts that are needed to characterize the starting monomers for the network formation with epoxy resin are the 


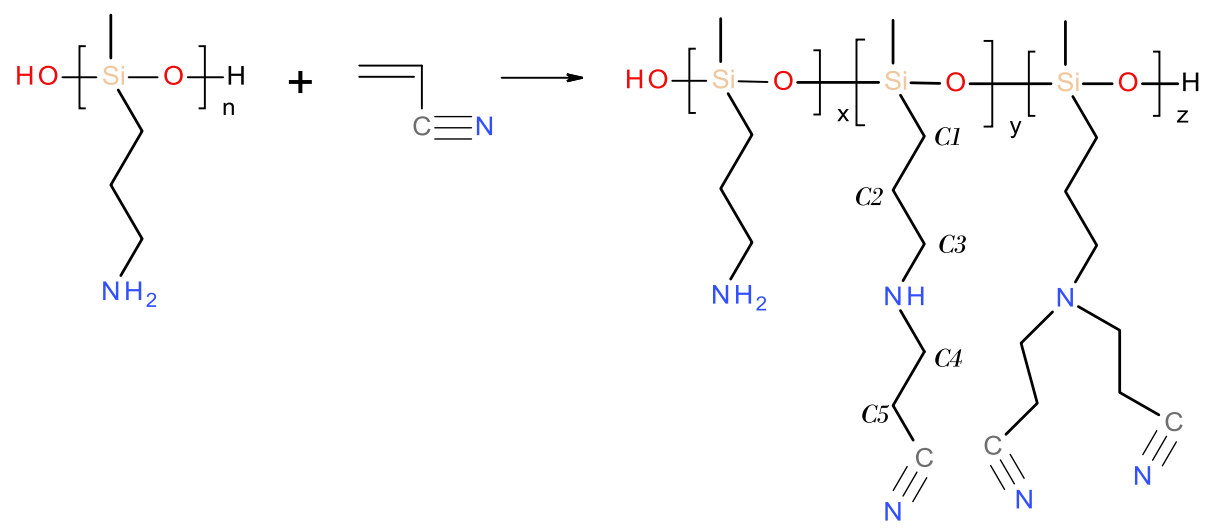

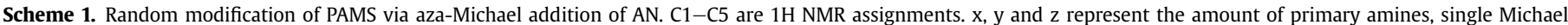
addition and double Michael addition respectively.

amount of AN effectively coupled, which determines the functionality of the adduct, and the presence of double additions (segment $\mathrm{z}$ in Scheme 1), which would create tertiary amines introducing dangling ends in the network.

${ }^{1} \mathrm{H}$ NMR was used to measure the extent of the coupling reaction Fig. 1 shows the ${ }^{1} \mathrm{H}$ NMR spectra for PAMS and some selected adducts; the peak labels are explained in Scheme 1.

Amine signals broaden, decrease in intensity and position of signals is moved to a lower value of the chemical shift as the extent of coupling increases. They are generally strongly affected by hydrogen bonding, concentration or even protonation being thus not adequate for quantification. The signals of protons C4 and C5 from the AN moiety appear around the signal of PAMS C3 protons. At all AN contents, C4 peak overlaps the C3 PAMS peak being not adequate for integration. Peak C5 (AN) and C1 and C2 (both from PAMS) are well resolved at low AN contents, but as AN content increases above $50 \%$, C5 tail overlaps with C3 tail being extremely difficult to separate the signals of both protons. Since our main interest is to ensure that addition reaction has reached total conversion, we have used two integration methods that overestimate (absolute integration) and underestimate (base line corrected integration) the signals of protons and calculated an average composition. Results are shown in Fig. 2 were the ratio AN: $\mathrm{NH}_{2}$ obtained from NMR integration is plotted against the feed molar ratio.
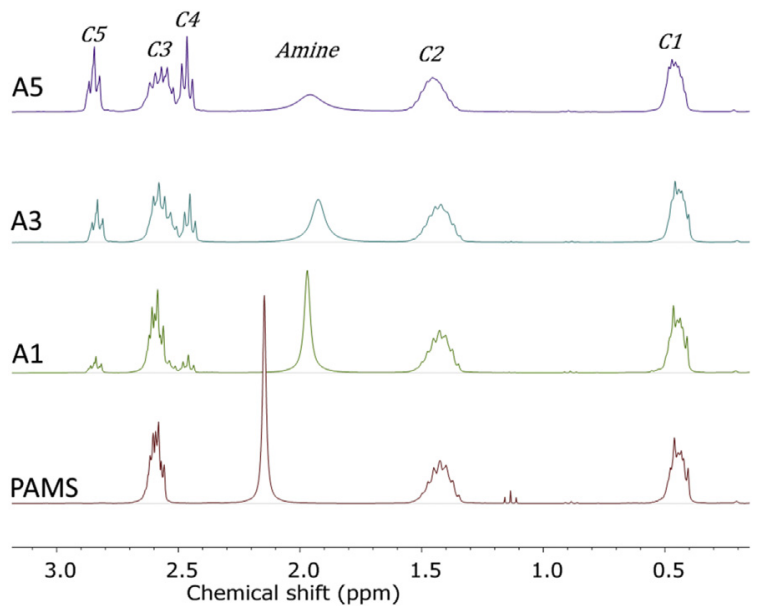

Fig. 1. ${ }^{1} \mathrm{H}$ NMR spectra of PAMS and some selected adducts. Peak labels are indicated in Scheme 1.
It is worth noting the extremely good correlation between both magnitudes which confirms that aza-Michael addition reached $100 \%$ conversion under our experimental conditions.

${ }^{1} \mathrm{H}$ NMR does not provide information about possible double additions, so we have also analyzed the FTIR spectra of all the samples. Fig. S2 (Supplementary material) shows the spectra in the mid-infrared range and Fig. S3a (Supplementary material) shows the spectra in the near range. In the mid-range, it can be clearly observed the gradual disappearance of the primary amine bending band $\left(1600 \mathrm{~cm}^{-1}\right)$ and the substitution of the two stretching bands at $3400-3300 \mathrm{~cm}^{-1}$ by a single band $\left(3400 \mathrm{~cm}^{-1}\right)$, which is characteristic of secondary amines [17]; in the near-range, the band appearing at $\sim 6500 \mathrm{~cm}^{-1}$, gradually changes its shape from 6534 $\mathrm{cm}^{-1}$, an overtone of primary amines, to $6479 \mathrm{~cm}^{-1}$, charac-teristic of secondary amines. These observations clearly suggest that single aza-Michael addition on PAMS primary amines has taken place in all cases, that is to say, no tertiary amines should be expected. For quantitative purposes we have analyzed the primary amine broad combination band centered at $4937 \mathrm{~cm}^{-1}$ which is easy to integrate (Fig. S3). However, this band shows a non-assigned high energy shoulder that gradually disappears with AN

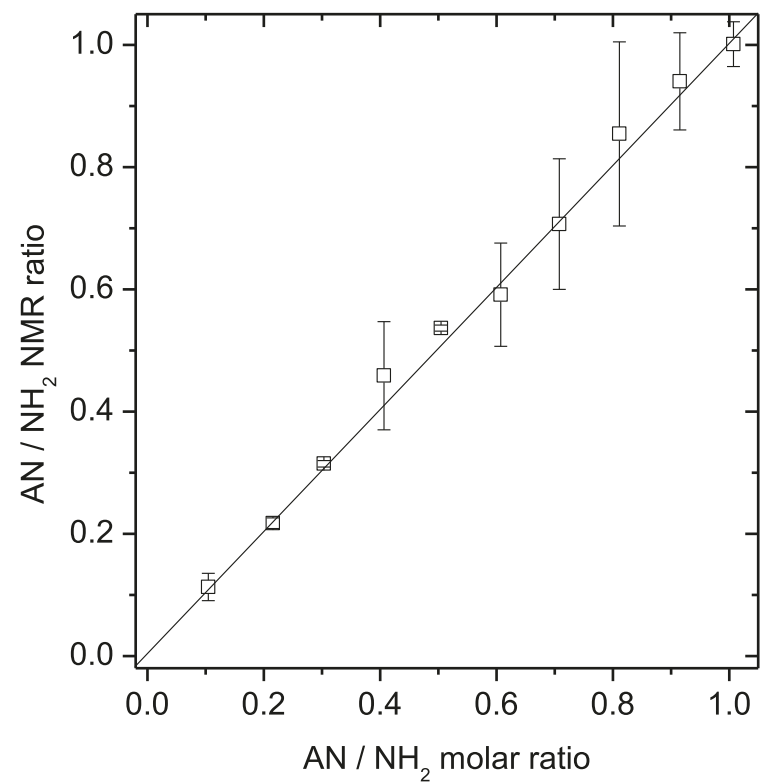

Fig. 2. $\mathrm{AN} / \mathrm{NH}_{2}$ ratio from $\mathrm{NMR}$ integration against the molar ratio used in the feed. Line is the diagonal. 
content. For a proper integration, only the low energy half portion of the band has been considered as shown in Fig S3b.

Results of the FTnIR integration are shown in the form of percentage of reacted primary amine as a function of initial concentration of $\mathrm{AN}$ in Fig. 3. Depending on the relative reactivity of primary and secondary amines, $R_{21}$, the extent of double Michael addition can be important especially at high AN contents. Although it is generally assumed that the reactivity of aliphatic secondary amines is higher than primary amines because they are more nucleophilic [12], it has been also reported that due to electronic or steric hindrance secondary amines may present low reactivity [18]. In our case, the nitrile acceptor group may inductively deactivate the secondary amine reducing its reactivity against Michael addition.

To ascertain whether single or double addition has taken place, we have simulated two realistic cases: i) secondary amine has very low reactivity, and ii) reactivity of primary and secondary amines is the same. In case i) the percentage of reacted amine is directly given by the $\mathrm{AN}: \mathrm{NH}_{2}$ ratio. For case ii) the percentage of reacted primary amine can be easily calculated from simple probabilistic arguments [19] as $100 \times\left[1-(1-1 / r)^{2}\right]$, where $r=2\left[\mathrm{NH}_{2}\right]_{0} /[A N]_{0}$. Both simulations are jointly represented in Fig. 3 where it can be clearly observed that experimental data coincide extremely well with case i). Thus, double addition is negligible under our experimental conditions and, consequently, the synthesized adducts do not contain any appreciable amount of tertiary amines, i.e. $z \approx 0$ in Scheme 1.

The molar mass and composition of the modified oligomers, as obtained from MALDI, NMR and FTnIR, are summarized in Table S1 (Supplementary material).

Glass transition temperatures of the adducts were measured by DSC and the results are presented in Fig. 4. The adducts were considered as copolymers of the two monomers shown in Scheme 1 ; monomer 1 containing the $-\mathrm{NH}_{2}$ functionality and monomer 2 containing the $-\mathrm{CN}$ functional group.

Glass transition temperatures of the random copolymers pre-sent positive deviations of the Flory-Fox behavior suggesting

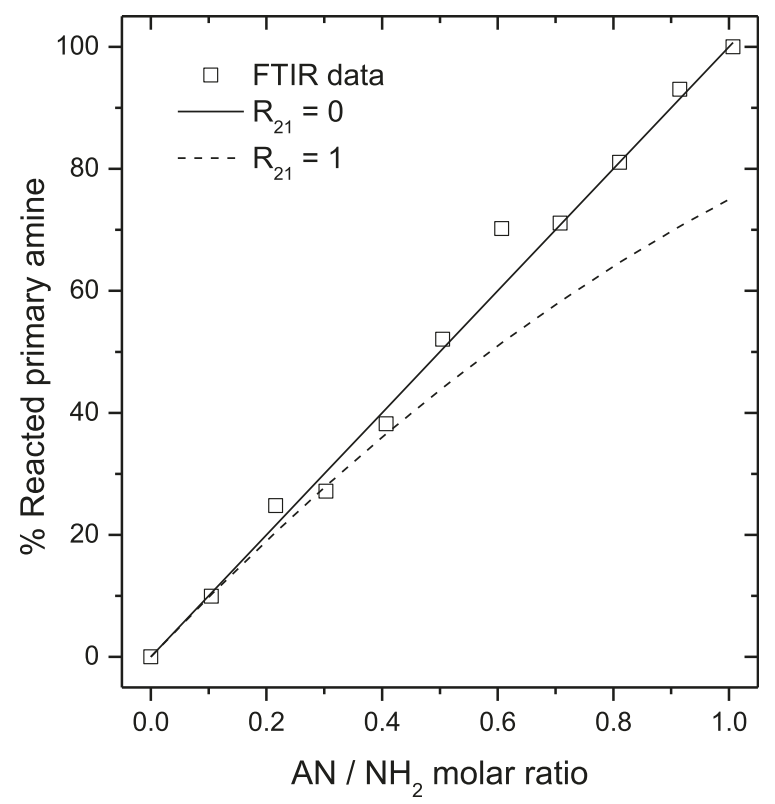

Fig. 3. Percentage of reacted primary amine as obtained from FTnIR as a function of the initial $\mathrm{AN} / \mathrm{NH}_{2}$ molar ratio in the feed. Continuous and dash dotted lines represent theoretical predictions assuming secondary to primary amine reactivity, $R_{21}$, equal to 0 or 1 respectively.

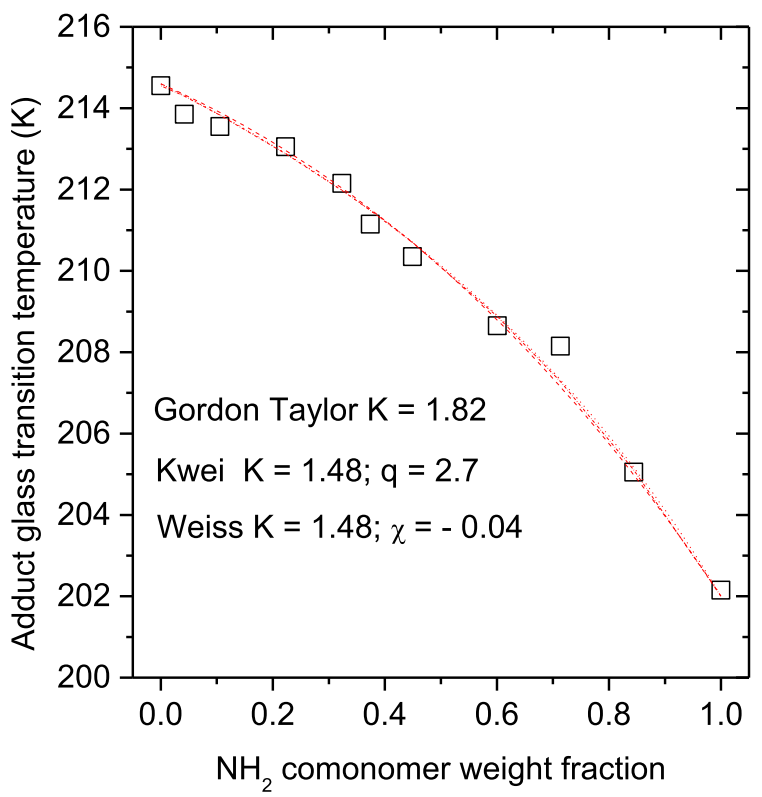

Fig. 4. Glass transition temperature of PAMS and adducts as a function of the weight fraction of $\mathrm{NH}_{2}$ comonomer in the copolymer. Data correspond to fitting parameters. Lines are best fits to Gordon Taylor, Kwei and Weiss models.

relatively strong intermolecular interactions. Data could be fitted to the Gordon Taylor equation leaving the constant $\mathrm{K}$ as a fitting parameter [20] giving a value of the fitting constant $\mathrm{K}_{0}=1.82$; values of $K_{0}>1$ are common in systems were there are attractive interactions [21]. However, since $K_{0}$ has unclear physical meaning we have fitted the data to other models that consider attractive interactions such as the Kwei equation [22] or the Weiss model [23]. In these models, the parameter $\mathrm{K}$ is defined as in the Couchman's model [24] $K_{C}=\Delta C_{p 1} / \Delta C_{p 2}$, i.e., the ratio between the heat capacity changes at the glass transition temperature of both polymers (PAMS and A10). The measured value of $K_{C}$ was 1.48 and it was used as a fixed constant. Kwei model yields a value of the $q$ constant of 2.7; Weiss model yields a slightly negative value of the Flor-y-Huggins interaction parameter. However, since Tg values vary in a narrow temperature range $(14 \mathrm{~K})$ both models predict very similar trends and differences are lower than experimental uncertainty in the $\mathrm{Tg}$ determination. It can be therefore concluded that adducts are self-associated probably due to weak hydrogen bonding be-tween $-\mathrm{NH}_{2}$, $-\mathrm{NHR}$ and $-\mathrm{CN}$ groups as well as by dipole-dipole interactions between $-\mathrm{CN}$ groups.

\subsection{Relaxations of the cured networks}

Stoichiometric mixtures of the well characterized adducts with DGEBA were cured as explained in the experimental part and the low and high temperature relaxations were analyzed by DMTA. Fig. $5 \mathrm{a}-\mathrm{b}$ shows the storage and loss moduli and the $\tan \delta$ variation of the cured specimens in the temperature range $25-200{ }^{\circ} \mathrm{C}$; $\tan \delta$ variation in the low temperature range is presented in Fig. 6.

\subsubsection{Low temperature relaxations}

Low temperature relaxations were analyzed in terms of the temperature variation of the loss tangent (Fig. 6), activation energies and peak temperatures (Fig. 7). PAMS/DGEBA network presents a broad relaxation $\left(\beta_{2}\right)$ centered at about $-50{ }^{\circ} \mathrm{C}$ (Fig. $5 \mathrm{c}$ ) which is attributed to a complex set of motions that involve both the hydroxypropylether group $\mathrm{O}-\mathrm{CH}_{2}-\mathrm{CHOH}-\mathrm{CH}_{2}$ and the DGEBA ring flips, as it is commonly found in epoxy resins [25]. The 


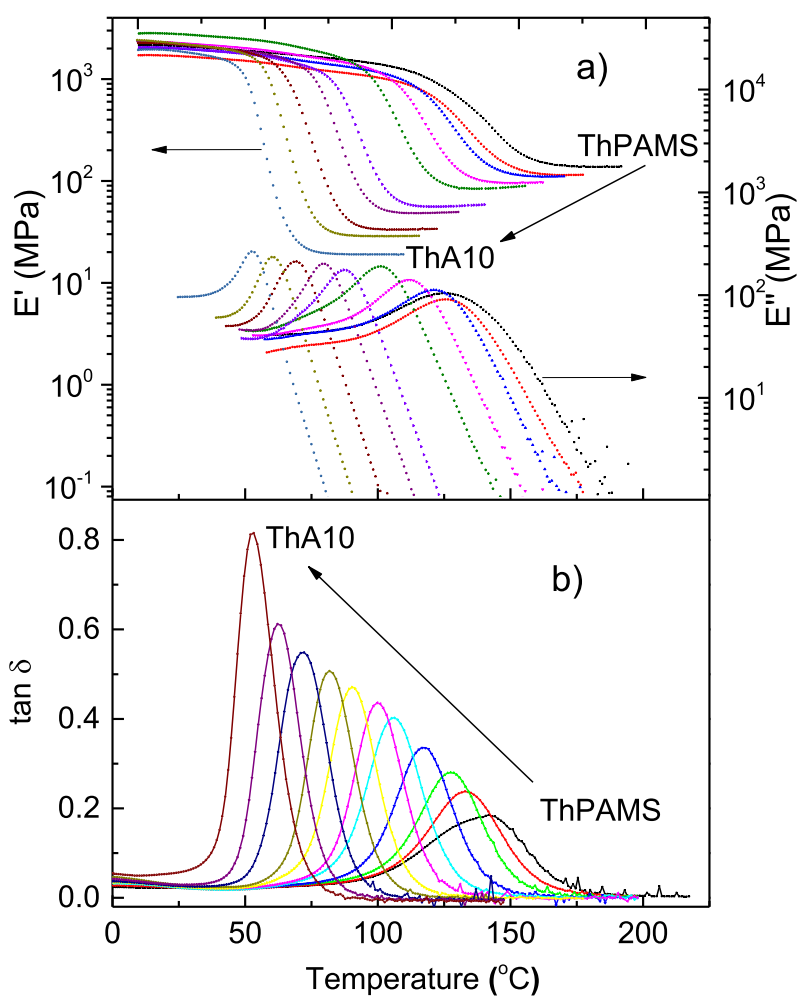

Fig. 5. Storage and loss moduli (a) and loss tangent at $1 \mathrm{~Hz}$ (b) for ThPAMS and the adducts cured with DGEBA, ThAx.

activation energy calculated from Arrhenius plots of $\tan \delta$ at 1,10 and $30 \mathrm{~Hz}$ (not shown) is $E_{a}\left(\beta_{2}\right)=65 \pm 4 \mathrm{~kJ} \mathrm{~mol}^{-1}$ (see Fig. 7) which is coincident with reported data for highly crosslinked epoxy systems [25].

On addition of acrylonitrile loss tangent decreases and $\beta_{2}$ peak clearly shifts to lower temperatures (Figs. 6 and 7). This result indicates that the amount of hydroxypropylether groups decreases becoming progressively more mobile; this is in accordance with the

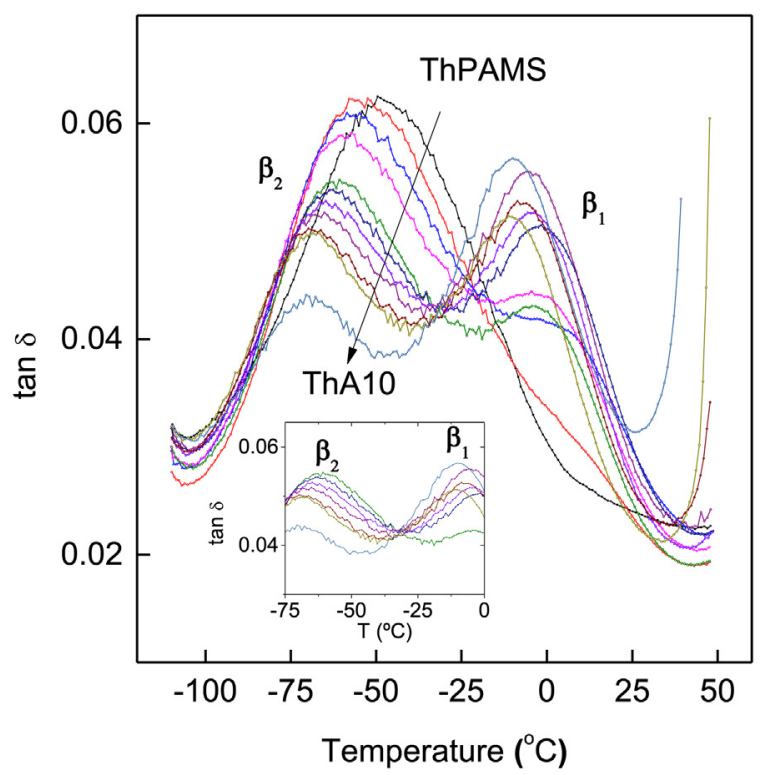

Fig. 6. Loss tangent at $1 \mathrm{~Hz}$ in the low temperature range for all specimens. Inset shows the isosbestic point linking $\beta_{1}$ and $\beta_{2}$ for cured adducts ThA $4-$ ThA10.

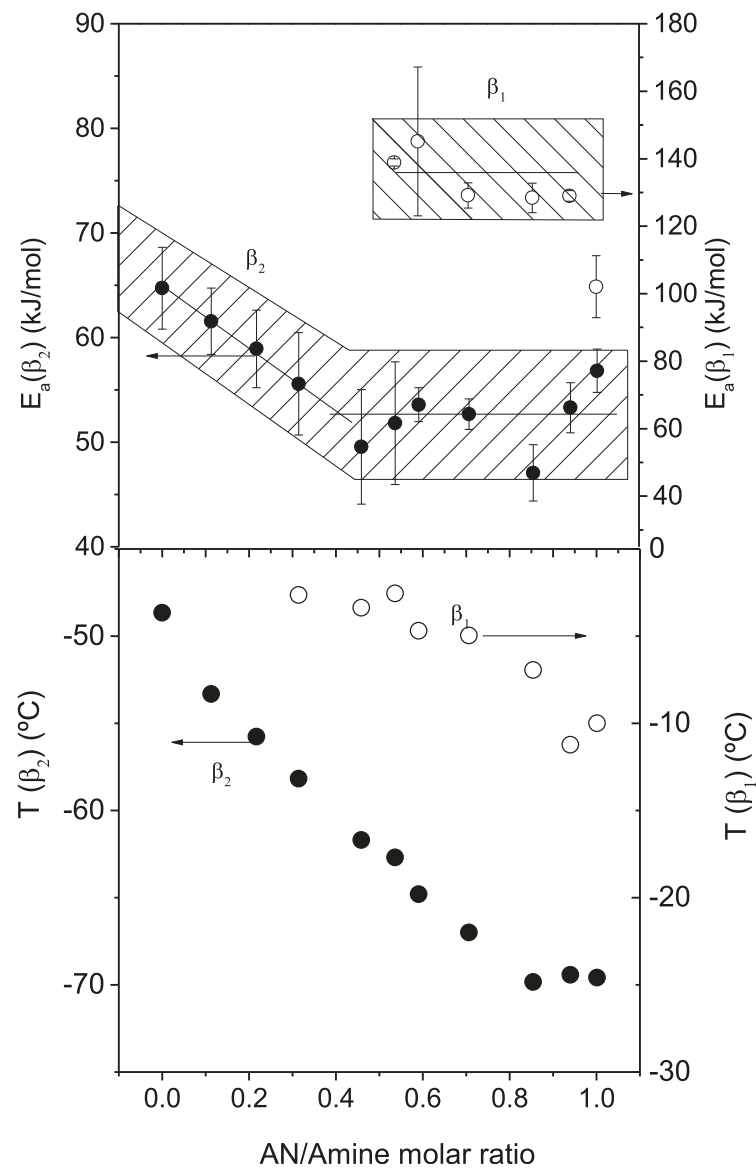

Fig. 7. Up: activation energies for the $\beta_{2}$ (left) and $\beta_{1}$ (right) relaxations as a function of the nitrile content. Error bars correspond to the fitting standard deviation. Lines and shadow areas are eye guides Bottom: peak temperatures for $\beta_{2}$ (left) and $\beta_{1}$ (right) relaxations.

two following facts: i) aza-Michael addition progressively lowers the amount of primary amine which lowers the amount of hydroxypropylether groups, and ii) amine functionalities stop being trifunctional crosslinks each time a nitrile is added to them, hence, crosslinking degree decreases.

Shifting of the $\beta_{2}$ transition to lower temperatures as crosslinking degree decreases has been already observed by Monnerie [25], among others [26], and should be followed by a reduction of the activation energy. This is observed in Fig. 7, where $E_{a}\left(\beta_{2}\right)$ is plotted as a function of nitrile molar ratio but only up to $40 \%$ nitrile. At higher nitrile contents activation energy seems to reach a constant value.

Interestingly, Fig. 6 shows that as nitrile content increases, a new relaxation $\left(\beta_{1}\right)$ appears shifted to higher temperatures, around $0{ }^{\circ} \mathrm{C}$. At low nitrile contents this relaxation overlaps with $\beta_{2}$ being impossible to determine both the peak temperatures and the activation energies but, as shown in Fig. 7, at nitrile contents higher than $\sim 50 \%$ peak temperatures seem to slightly decrease while $E_{a}\left(\beta_{1}\right)$ seems to be constant around the value of $139 \mathrm{~kJ} \mathrm{~mol}^{-1}$. It should be noted also that at these high nitrile contents $E_{a}\left(\beta_{2}\right)$ also remains almost constant around the value of $52 \mathrm{~kJ} \mathrm{~mol}^{-1}$.

The origin of this new relaxation is not straightforward. Since the intensity of this relaxation grows with nitrile content a paral-lelism with the $\beta$ relaxation of polyacrylonitrile (PAN) seems to be plausible. However, this last relaxation appears at 330-340 $\mathrm{K}$ in PAN, its activation energy is around $110 \pm 10 \mathrm{~kJ} \mathrm{~mol}^{-1}$ and involves 8 monomeric units [27]. 
Fortunately, detailed inspection of Fig. 6 reveals the presence of an isosbestic point at $-31{ }^{\circ} \mathrm{C}$ linking $\beta_{2}$ and $\beta_{1}$ relaxations for thermosets ThA4 - ThA10. To the authors knowledge, isosbestic points in the mechanical relaxations of amorphous polymers are uncommon although they have been observed in the dielectric relaxations of partially crystalline polymers $[28,29]$. The presence of an isosbestic point suggests that the new $\beta_{1}$ relaxation grows at the expense of $\beta_{2}$, i.e. as nitrile content increases the $\beta_{2}$ relaxation transforms into the $\beta_{1}$. This is verified by the constancy of the sum of both loss tangents (not shown). In addition, since $\beta_{1}$ has a notably higher activation energy, the number of atoms involved in the relaxation must be also higher [27], hence, it should involve a more extended region probably comprising the hydroxypropylether group, the tertiary amine, the nitrile side chain and the propyl chain that connects the whole ensemble with the polyorganosiloxane cyclic or linear oligomers. This proposed region is illustrated in Scheme 2.

Why the isosbestic point is not observed for samples ThA1 ThA3 may be associated to some degree of intermolecular cooperativity which gradually disappears as hydroxypropylether moiety becomes progressively isolated or diluted in the network, although it should not be discarded an effect of the characteristic heterogeneity of these samples.

\subsubsection{High temperature relaxations, $T_{\alpha}$ and rubber modulus}

In the high temperature range, networks present a single well defined relaxation associated to the $\alpha$ relaxation (Fig. 5a, b) except for the PAMS and ThA1 systems, where loss tangent is broad and probably reflects some kind of heterogeneity due to the initial poor miscibility of DGEBA with PAMS and with low nitrile adducts. It is known that DGEBA-PAMS system undergoes reactive compatibilization using a simple one step curing protocol at high curing temperatures; however, some network heterogeneities remain resulting in a broadening of the loss tangent [9], although other authors [7] obtained a single well defined peak using a different curing protocol. To verify if nitrile moieties help in compatibilizing the hardener with DGEBA, at least in the micron range, PAMS and the adducts were doped with 4-amino-1,8-naphthalic anhydride, a fluorescent label, and images of the curing systems were taken by laser scanning confocal microscopy in the postgel stage after curing at low temperatures $\left(50{ }^{\circ} \mathrm{C}\right)$. In Fig. 8 we present those images, where the bright regions correspond to PAMS or adduct rich domains. For networks with PAMS and adducts $A 1-A 3$, the observed morphology clearly reflects immiscibility of the components at low temperatures; however, as nitrile content increases, both components progressively become more miscible, and for adducts A5 - A10, the networks appear completely miscible.

Therefore, it seems that there is a relationship between the miscibility of both components and the narrowing of the loss tangent peak for the $\alpha$ relaxation (see Table 1 ), which is commonly associated with the spatial distribution of crosslinking density $[19,30]$. A recent work on double epoxy networks also shows a broadening in tan $\delta$ compared with unimodal networks [31].

Another interesting effect of the nitrile incorporation into the network is the increment of the loss tangent and, specifically for ThA10, the notably high value it achieves at temperatures near room temperature. Incorporation of flexible nitrile side chains do not contribute to the elasticity of the network but provide a frictional pathway for dissipating mechanical work. The high values of loss tangent are similar to those presented by the so-called superdamping elastomers, which range between 0.5 and 0.8 .

Glass transition temperatures were determined from the $\alpha$ relaxation loss tangent peaks and Young's modulus in the rubbery state was obtained at $T_{\alpha}+50 \mathrm{~K}$; both data appear in Table 1 . Rubber moduli for DGEBA epoxy - aliphatic amine resins are usually found in the order of $\sim 50 \mathrm{MPa}$ depending on crosslink density and network structure. This is the case of the early reported data on linear polyamines [32] (DETA, TETA, TEPA). The very high values found for the systems studied here have not been found in the literature; only tetrafunctional epoxies cured with anhydrides present exceedingly higher modulus values [33]. However, using branched aliphatic amine hardeners of functionality $f>4$ or quasidendrimers (for example, $\mathrm{N}, \mathrm{N}, \mathrm{N}^{\prime}, \mathrm{N}^{\prime}, \mathrm{N}^{\prime \prime}$-penta(3-aminopropyl)diethylenetriamine), a noticeable increase in modulus up to $\sim 70 \mathrm{MPa}$, which cannot be related only to an increased crosslinking density, has been achieved [34,35]. The concentration of elastically active chains (EANC), $v$, has been calculated from the rubbery modulus and is presented in Table 1.

As it is commonly found in epoxy thermosets reduction of crosslinking degree is accompanied by a decrease in $T_{\alpha}$. Crosslinks reduce configurational entropy as well as the free volume and the frequently used models that account for these effects are those of DiMarzio [36], DM, and Fox-Loshaek [37], F-L.

The basic equations for F-L and DM models are given by Eqs. (1) and (2) respectively.

$T_{g}=T_{g, l}+K_{F L} \nu$

$T_{g}=T_{g, l} /\left(1-K_{D M} F \cdot \nu\right)$

$T_{g}$ is the glass transition temperature of a network having $\nu$

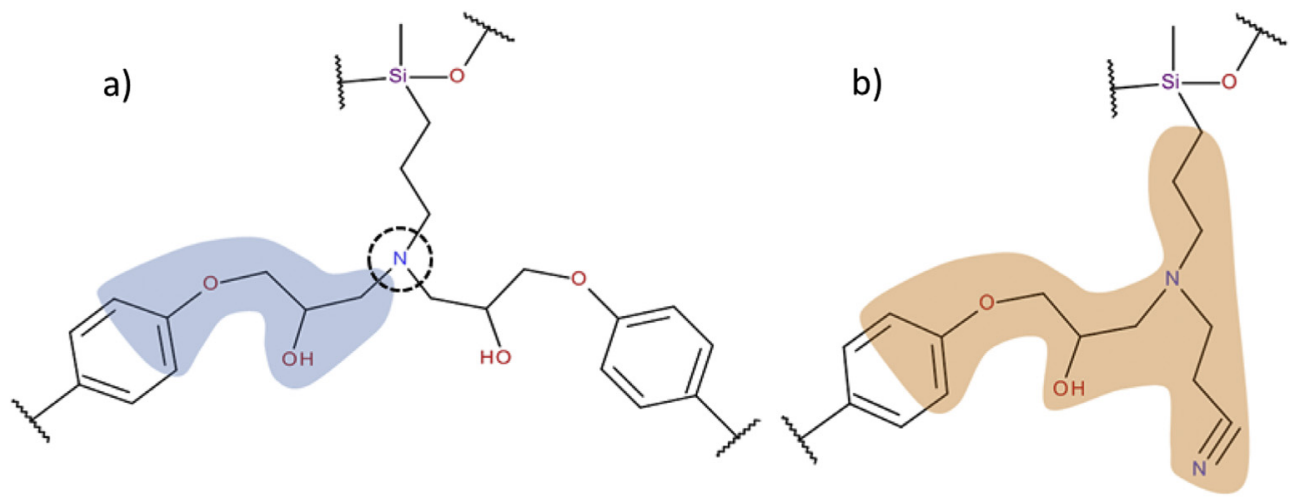

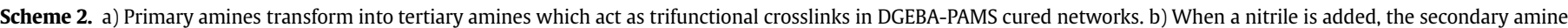

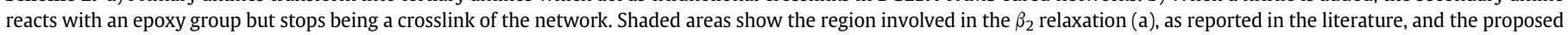
region for the $\beta_{1}$ relaxation (b). 

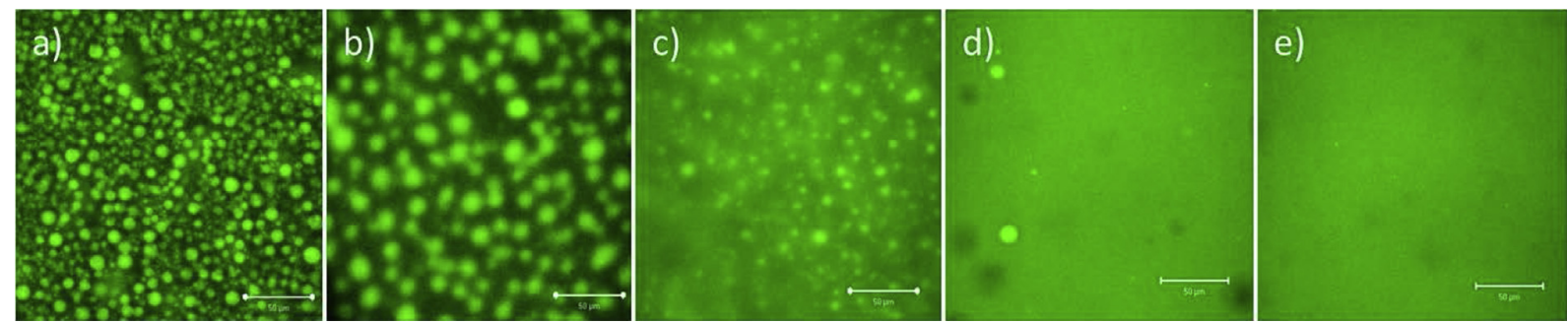

Fig. 8. Laser scanning confocal microscopy images of systems a) ThPAMS, b) ThA1, c) ThA3, d) ThA5 and e) ThA7. Scale bar is 50 microns.

elastically active chains ( $\mathrm{mol} \mathrm{kg} \mathrm{kg}^{-1}$ ); $T_{g, l}$ is the glass transition temperature of a linear polymer with the same composition as the network; $F$ is the flex parameter $\left(\mathrm{g} \mathrm{mol}^{-1}\right)$ that accounts for the chain stiffness; $K_{F L}$ and $K_{D M}$ are the F-L (in $\mathrm{K} \mathrm{g} \mathrm{mol}^{-1}$ ) and DM constants respectively.

The use of these models requires the knowledge of the elastically active chain concentration (EANC) which can be obtained from a model of the network structure. In conventional thermosetting systems knowledge of this structure is straightforward assuming that tertiary amines are tri-functional crosslinks. In our hybrid systems, in addition to tertiary amines we have silicon atoms from the cyclics fraction, which may participate as trifunctional crosslinks, and the non-terminal silicon atoms from the linear fraction, which also may participate as trifunctional crosslinks (see Scheme 2). However, two of the three chains emerging from a silicon atom consist of a single oxygen atom and it appears to be quite unreasonable to consider them as elastic chains. Alternatively, polysiloxane chain and cyclic molecules can be considered as crosslinks as a whole, but EANC data derived from this model (not shown) are far from those obtained experimentally (Table 1). A paper dealing with this disagreement is under preparation but for the present discussion we have explored the use of $\nu$ calculated from elasticity measurements.

Fitting to F-L and DM models is presented in Fig. 9. Although current precision in the measurement of the relaxa-tion temperature and Young's moduli do not allow to ascertain which model better reflects the behavior of our systems, the values of the fitting parameters are coincident with data for similar sys-tems and have physical sense. For example $T_{g, l}=312-319 \mathrm{~K}$ is a reasonable value for a linear analogue of the chains between crosslinks, and the flex parameter $\mathrm{F} \sim 12 \mathrm{~g} \mathrm{~mol}^{-1}$ (assuming $K_{D M} \sim 2$ as it is commonly reported in the literature [19]) is very similar to data for fully aliphatic systems containing flexible bonds [38](-O-).

\section{Table 1}

Density, glass transition temperature, breath of the relaxation, Young's modulus in the rubbery state and concentration of elastically active chains.

\begin{tabular}{llllll}
\hline Sample & $\rho^{\mathrm{a}} \mathrm{kg} \mathrm{m} \mathrm{[3]}$ & $T_{\alpha}{ }^{\circ} \mathrm{C}$ & $\Delta \tan \delta^{\mathrm{b}}{ }^{\circ} \mathrm{C}$ & $E^{\mathrm{C}} \mathrm{MPa}$ & $v^{\mathrm{d}} \mathrm{mol} \mathrm{kg}^{-1}$ \\
\hline ThPAMS & 1170 & 141.9 & 45.7 & 138 & 10.55 \\
ThA1 & 1159 & 133.0 & 34.1 & 117 & 9.00 \\
ThA2 & 1169 & 127.5 & 29.6 & 114 & 8.73 \\
ThA3 & 1150 & 117.2 & 26.0 & 101 & 7.87 \\
ThA4 & 1159 & 106.3 & 25.5 & 92 & 7.02 \\
ThA5 & 1150 & 99.8 & 22.3 & - & - \\
ThA6 & 1187 & 90.3 & 21.3 & 60 & 4.73 \\
ThA7 & 1188 & 81.7 & 20.5 & 51 & 4.17 \\
ThA8 & 1191 & 72.0 & 21.1 & 34 & 2.93 \\
ThA9 & 1191 & 62.6 & 19.2 & 29 & 2.58 \\
ThA10 & 1197 & 53.2 & 17.0 & 19 & 1.74 \\
\hline
\end{tabular}

${ }^{\text {a }}$ Measured at $35.1^{\circ} \mathrm{C}$.

b Measured at half width.

c Rubber modulus measured at $\mathrm{Tg}+50{ }^{\circ} \mathrm{C}$.

d calculated from Young's moduli according to $v=E_{r u b} / 3 R T \rho$.
Therefore, thermal relaxations are coherent with elastic behavior since the later helps to explain the former, but the question about the network model that supports the experimental concentration of elastically active chains needs a deeper thought.

\section{Conclusions}

A mixture of linear and cyclics of PAMS, well characterized by MALDI, was modified with acrylonitrile via aza-Michael addition yielding oligomers with controlled primary: secondary amine ratios and with an improved miscibility with DGEBA epoxy resin (for AN: $\mathrm{NH}_{2}$ ratios higher than 0.4 ). Incorporation of nitrile groups induces positive deviations of $\mathrm{Tg}$ as the nitrile content increases, and fitting to Gordon Taylor, Kwei and Weiss equations revealed weak attractive interactions between pendant groups.

A broad range of organo-inorganic thermosets were prepared with $\mathrm{AN}: \mathrm{NH}_{2}$ ratios ranging between 0 and 1 . The low temperature mechanical behavior shows a single relaxation for ThPAMS, $\beta_{2}$, which was attributed to the hydroxypropylether moiety as it is commonly found in epoxy systems. However, as nitrile content was increased an additional relaxation appeared at higher temperatures, $\beta_{1}$, with a higher activation energy. It was found that both relaxations were linked by an isosbestic point which was explained assuming that the molecular segment associated to $\beta_{1}$ was longer and included that for $\beta_{2}$ relaxation.

Modulus of elasticity at small deformations in the plateau regime was measured by DMTA giving very high values, even higher than any reported epoxy system cured with amines. Consequently, EANC derived from experiments were very high and no rational model based on compositional analysis could account for this disagreement. To explain the high T relaxations common

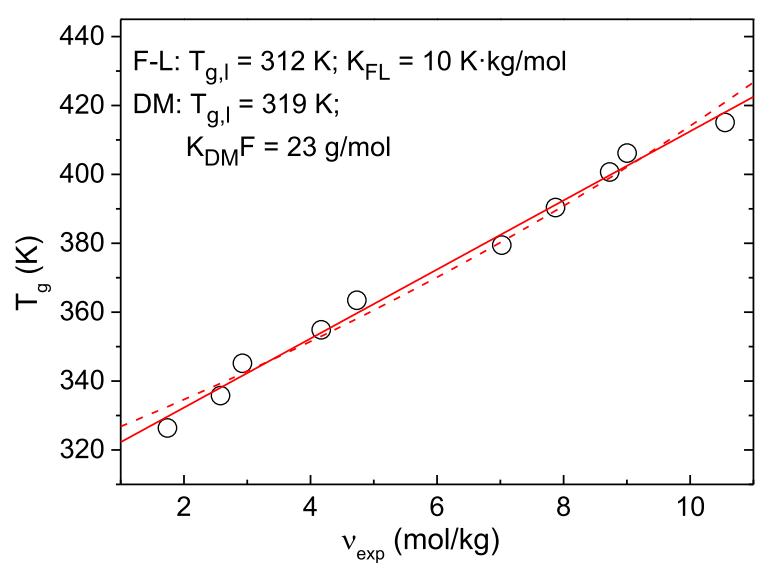

Fig. 9. Variation of the glass transition temperature as a function of the concentration of elastically active chains calculated from elasticity measurements. Continuous line corresponds to fitting to the Fox-Loshaek equation; dashed line corresponds to the DiMarzio equation fit. Fitting parameters are indicated in the inset. 
structure-Tg relationships were used along with the experimentally determined EANC from DMTA and reasonable fitting parameters were obtained.

\section{Acknowledgment}

Authors wish to thank Spanish Ministerio de Economía y Competitividad for funding this work under grant MAT2010-17091.

\section{References}

[1] J.J. Chruściel, E. Leśniak, Modification of epoxy resins with functional silanes, polysiloxanes, silsesquioxanes, silica and silicates, Prog. Polym. Sci. 41 (2015) 67-121

[2] M. Srividhya, M.S. Lakshmy, B.S.R. Reddy, Chemistry of siloxane amide as a new curing agent for epoxy resins: material characterization and properties, Macromol. Chem. Phys. 206 (2005) 2501-2511.

[3] E. Yilgör, I. Yilgör, Silicone containing copolymers: synthesis, properties and applications, Prog. Polym. Sci. 39 (2014) 1165-1195.

[4] S.-S. Hou, Y.-P. Chung, C.-K. Chan, P.-L. Kuo, Function and performance of silicone copolymer. Part IV. Curing behavior and characterization of epox$\mathrm{y}$-siloxane copolymers blended with diglycidyl ether of bisphenol-A, Polymer 41 (2000) 3263-3272.

[5] J.C. Cabanelas, B. Serrano, J. Gonzalez-Benito, J. Bravo, J. Baselga, Morphology of epoxy/polyorganosiloxane reactive blends, Macromol. Rapid Commun. 22 (2001) 694-699.

[6] J.C. Cabanelas, B. Serrano, M.G. González, J. Baselga, Confocal microscopy study of phase morphology evolution in epoxy/polysiloxane thermosets, Polymer 46 (2005) 6633-6639.

[7] M. Gonzalez, P. Kadlec, P. Štěpánek, A. Strachota, L. Matějka, Crosslinking of epoxypolysiloxane system by reactive blending, Polymer 45 (2004) 5533-5541.

[8] M. González, Termoestables híbridos basados en resinas epoxi hidrogenadas y polisiloxanos: curado, morfología y modificación con HTPB y PMMA, PhD Thesis, Universidad Carlos III, Madrid, 2008

[9] J.C. Cabanelas, B. Serrano, J. Baselga, Development of co-continuous morphologies in initially heterogeneous thermosets blended with PMMA, Macromolecules 38 (2005) 961-970.

[10] J.C. Cabanelas, S.G. Prolongo, B. Serrano, J. Bravo, J. Baselga, Water absorption in polyaminosiloxane-epoxy thermosetting polymers, J. Mat. Proc. Tech. 143-144 (2003) 311-315.

[11] S.G. Prolongo, J.C. Cabanelas, J. Baselga, Reactive compatibilization of epoxy/ polyorganosiloxane blends, Macromol. Symp. 198 (2003) 283-293.

[12] B.D. Mather, K. Viswanathan, K.M. Miller, T.E. Long, Michael addition reactions in macromolecular design for emerging technologies, Prog. Polym. Sci. 31 (2006) 487-531.

[13] J.C. Cabanelas, Polisiloxanos reactivos. Agentes de compatibilización y curado, $\mathrm{PhD}$ Thesis, Universidad Carlos III de Madrid, 2000.

[14] E. Dermitzaki, B. Wunderle, J. ;Bauer, H. Walter, B. Michel, Structure Property correlation of epoxy resins under the influence of moisture and comparison of diffusion coefficient with MD-simulations, in: Proceedings of the IEEE 9th. Int. Conf on Thermal, Mechanical and Multiphysics Simulation and Experiments in MicroElectronics and Micro-Systems, EuroSimE 2008, Freiburg-im-Breisgau, Germany, April 2008.
[15] M.B. Smith, Methods of Non- $\alpha$-amino Acid Synthesis, second ed., CRC Press, Boca Raton, 2013, p. 65.

[16] C. Mukherjee, A.K. Misra, Lett. Org. Chem. 4 (2007) 54-59.

[17] M. González, J.C. Cabanelas, J. Baselga, in: T. Theophanides (Ed.), Infrared Spectroscopy - Materials Science, Engineering and Technology, In-Tech, 2012. Chap. 13

[18] D. Wang, Y. Liu, Z. Hu, C. Hong, C. Pan, Michael addition polymerizations of trifunctional amines with diacrylamides, Polymer 46 (2005) 3507-3514.

[19] J.P. Pascault, H. Sautereau, J. Verdu, R.J.J. Williams, Thermosetting Polymers, Marcel Dekker Inc., New York, 2002, p. p 85.

[20] H.A. Schneider, J. Rieger, E. Penzel, The glass transition temperature of random copolymers: 1 . Experimental data and the Gordon-Taylor equation, Polymer 38 (1997) 325-337.

[21] M. Aubin, R.E. Prud'homme, Analysis of the glass transition temperature of miscible polymer blends, Macromolecules 21 (1988) 2945-2949.

[22] T.K. Kwei, The effect of hydrogen bonding on the glass transition tempera-tures of polymer mixtures, J. Polym. Sci. Polym. Lett. 22 (1984) 307-313.

[23] X. Lu, R.A. Weiss, Relationship between the glass transition temperature and the interaction parameter of miscible binary polymer blends, Macromolecules 25 (1992) 3242-3246

[24] P.R. Couchman, Compositional variation of glass-transition temperatures. 2. application of the thermodynamic theory to compatible polymer blends, Macromolecules 11 (1978) 1156-1161.

[25] L. Heux, J.L. Halary, F. Lauprêtre, L. Monnerie, Dynamic mechanical and $13 \mathrm{C}$ n.m.r. investigations of molecular motions involved in the $\beta$ relaxation of
epoxy networks based on DGEBA and aliphatic amines, Polymer 38 (1997) epoxy networks

[26] J.-F. Shi, P.T. Inglefield, A.A. Jones, M.D. Meadows, Sub-glass transition motions in linear and cross-linked bisphenol-type epoxy resins by deuterium line shape NMR, Macromolecules 29 (1996) 605-609.

[27] a) V.A. Bershtein, V.M. Egorov, Differential Scanning Calorimetry of Polymers, Ellis Horwood, Chichester, 1994, p. 20; b) V.A. Bershtein, V.A. Ryzhov, Adv. Polym. Sci. 114 (1994) 93-121.

[28] F. Kremer, A. Schonhals, Broadband Dielectric Spectroscopy, Springer, Berlin, 2003.

[29] S. Cerveny, P. Zinck, M. Terrier, S. Arrese-Igor, A. Alegría, J. Colmenero, Dynamics of amorphous and semicrystalline 1,4-trans-poly(isoprene) by dielectric spectroscopy, Macromolecules 41 (2008) 8669-8676.

[30] A.T. Detwiler, A.J. Lesser, Characterization of double network epoxies with tunable compositions, J. Mater. Sci. 47 (2012) 3493-3503.

[31] I.M. McAninch, G.R. Palmese, J.L. Lenhart, J.J. La Scala, Characterization of epoxies cured with bimodal blends of polyether amines, J. Appl. Polym. Sci. 130 (2013) 1621-1631.

[32] D. Katz, A.V. Tobolsky, Rubber elasticity in a highly crosslinked epoxy system, Polymer 4 (1963) 417-421.

[33] P. Guerrero, K. De la Caba, A. Valea, M.A. Corcuera, I. Mondragon, Influence of cure schedule and stoichiometry on the dynamic mechanical behaviour of tetrafunctional epoxy resins cured with anhydrides, Polymer 37 (1996) 2195-2200.

[34] J. Wan, Z.-Y. Bu, C.-J. Xu, B.-G. Li, H. Fan, Preparation, curing kinetics, and properties of a novel low-volatile starlike aliphatic-polyamine curing agent for epoxy resins, Chem. Eng. J. 171 (2011) 357-367.

[35] J. Wan, C. Li, Z.-Y. Bu, C.-J. Xu, B.-G. Li, H. Fan, A comparative study of epoxy resin cured with linear diamine and a branched polyamine, Thermochim. Acta 188 (2012) 160-172.

[36] E.A. DiMarzio, On the second-order transition of a rubber, J. Res. NBS 68A (1964) 611-617.

[37] T.G. Fox, S. Loshaek, Influence of molecular weight and degree of crosslinking on the specific volume and glass temperature of polymers, J. Polym. Sci. 15 (1955) 371-390.

[38] V. Bellenger, J. Verdu, E. Morel, J. Polym, Effect of structure on glass transition temperature of amine crosslinked epoxies, Sci. B Polym. Phys. 25 (1987) 1219-1234. 